\title{
Hepatitis C Virus NS2 Protein Suppresses RNA Interference in Cells
}

\author{
Hui Zhou ${ }^{1} \cdot$ Qi Qian ${ }^{1,2} \cdot$ Ting $S^{2,3} \cdot$ Jiuyue $\mathrm{Xu}^{2,4} \cdot$ Jing Kong ${ }^{2,4} \cdot$ Jingfang $\mathrm{Mu}^{2} \cdot$ Yang $\mathrm{Qiu}^{2,4}$ (D) \\ Xi Zhou ${ }^{1,2,4}$ (i)
}

Received: 12 October 2019/Accepted: 1 November 2019/Published online: 27 November 2019

(c) Wuhan Institute of Virology, CAS 2019

\begin{abstract}
RNAi interference (RNAi) is an evolutionarily conserved post-transcriptional gene silencing mechanism and has been well recognized as an important antiviral immunity in eukaryotes. Numerous viruses have been shown to encode viral suppressors of RNAi (VSRs) to antagonize antiviral RNAi. Hepatitis C virus (HCV) is a medically important human pathogen that causes acute and chronic hepatitis. In this study, we screened all the nonstructural proteins of HCV and found that HCV NS2 could suppress RNAi induced either by small hairpin RNAs (shRNAs) or small interfering RNAs (siRNAs) in mammalian cells. Moreover, we demonstrated that NS2 could suppress RNAi via its direct interaction with doublestranded RNAs (dsRNAs) and siRNAs, and further identified that the cysteine 184 of NS2 is required for the RNAi suppression activity through a serial of point mutation analyses. Together, our findings uncovered that HCV NS2 can act as a VSR in vitro, thereby providing novel insights into the life cycle and virus-host interactions of HCV.
\end{abstract}

Keywords Hepatitis C virus (HCV) · NS2 · Antiviral RNAi · Viral suppressor of RNAi (VSR)

\section{Introduction}

$\mathrm{HCV}$ is a positive-sense, single-stranded RNA virus that belongs to the genus Hepacivirus of the family Flaviviridae. Its genome is approximately $9.6 \mathrm{~kb}$ in length and encodes a polyprotein that is cleaved by host and viral proteases to produce ten mature viral proteins, including structural core protein, envelope proteins E1 and E2, the p7

Electronic supplementary material The online version of this article (https://doi.org/10.1007/s12250-019-00182-5) contains supplementary material, which is available to authorized users.

Yang Qiu

yangqiu@wh.iov.cn

$\triangle$ Xi Zhou

zhouxi@wh.iov.cn

1 State Key Laboratory of Virology, College of Life Sciences, Wuhan University, Wuhan 430072, China

2 State Key Laboratory of Virology, Wuhan Institute of Virology, Center for Biosafety Mega-Science, Chinese Academy of Sciences, Wuhan 430071, China

3 Center for Translational Medicine, Wuhan Jinyintan Hospital, Wuhan 430040, China

4 University of Chinese Academy of Sciences, Beijing 100049, China ion channel protein, and nonstructural (NS) proteins NS2, NS3, NS4A, NS4B, NS5A and NS5B (Yin et al. 2018). $\mathrm{HCV}$ infection causes acute and chronic hepatitis in humans with a high propensity for chronicity. A large number of $\mathrm{HCV}$-infected patients fail to clear this virus and remain asymptomatic for years to develop severe liver diseases such as cirrhosis and hepatocellular carcinoma (Paboriboune et al. 2018). The capability of establishing chronic infection by $\mathrm{HCV}$ is partly due to its ability to evade host innate immune responses (Horner and Gale 2013).

RNAi is an evolutionarily conserved mechanism in all eukaryotes for post-transcriptional gene silencing. RNAi serves as an important antiviral immune response in a wide range of organisms, including fungi, plants, and invertebrates (Guo et al. 2019; Maillard et al. 2019). Moreover, recent studies have demonstrated that RNAi also exerts antiviral effects in mammals (Li et al. 2013; Maillard et al. 2013; Li et al. 2016; Qiu et al. 2017; Xu et al. 2019). The antiviral RNAi is triggered by the viral replicative intermediate double-stranded RNAs (vRI-dsRNAs) produced in the course of viral replication. These vRI-dsRNAs can be recognized and cleaved by host endoribonuclease Dicer into virus-derived small interfering RNAs (vsiRNAs) of approximately 21-23 nucleotide (nt). Argonaute (AGO) protein within RNA-induced silencing complexes (RISCs) 
incorporates one of the strands of vsiRNA duplex, which directs the RISC-mediated degradation of cognate viral RNAs (Maillard et al. 2019).

To counteract the antiviral RNAi, viruses have evolved to encode viral suppressors of RNAi (VSRs) targeting various steps of the RNAi pathway (Wu et al. 2010). One common strategy utilized by VSRs, including Nodamura virus (NoV) B2, Ebola virus (EBOV) VP35, influenza A virus (IAV) NS1, and human enterovirus 71 (EV-A71) 3A, is to prevent viral dsRNA from cleavage by Dicer ( $\mathrm{Li}$ et al. 2004; Sullivan and Ganem 2005; Haasnoot et al. 2007; Qiu et al. 2017). Alternatively, multiple VSR proteins have been shown to directly target the key components of the RNAi pathway, such as Dicer or AGO proteins. For example, Wuhan nodavirus (WhNV) B2 can directly bind to Drosophila Dicer-2 to inhibit vsiRNA production (Qi et al. 2011, 2012), while Cricket paralysis virus 1A has been found to directly inhibit Drosophila AGO2 (Nayak et al. 2018).

In the case of $\mathrm{HCV}$, the core protein was found to suppress RNAi by interacting with Dicer (Chen et al. 2008). Moreover, HCV structural E2 protein was also identified to antagonize RNAi by targeting AGO2 (Ji et al. 2008). Consistent with the findings in HCV, a number of RNA viruses were shown to encode more than one viral protein to counteract RNAi. For instance, VP35, VP30 and VP40 of EBOV were found to suppress RNAi through different mechanisms (Haasnoot et al. 2007; Fabozzi et al. 2011). Severe acute respiratory syndrome coronavirus (SARS-CoV) encoded 7a and nucleocapsid contain RNAi suppression activities (Karjee et al. 2010; Cui et al. 2015). Besides, Tat and Nef proteins of human immunodeficiency virus-1 (HIV-1) were identified to suppress RNAi through the dsRNA-binding and AGO interaction, respectively (Bennasser et al. 2005; Aqil et al. 2013). The phenomena of viruses encoding multiple VSRs as well as VSRs targeting different steps of the RNAi pathway highlight the importance of suppressing RNAi during the viral life cycle. Although the structural core and E2 proteins of $\mathrm{HCV}$ were shown to suppress RNAi, it is unclear if any HCV nonstructural protein contains RNAi suppression activity.

In this study, we uncovered that HCV nonstructural NS2 protein possessed a potent in vitro VSR activity that suppressed the RNAi induced by short hairpin RNA (shRNA) and siRNA in mammalian cells. We also confirmed the conserved residue in HCV NS2 that could disrupt its activity to suppress RNAi. Overall, our findings demonstrated that HCV NS2 contains VSR activity, thereby providing insights into the life cycle of $\mathrm{HCV}$.

\section{Materials and Methods}

\section{Plasmids and RNAs}

For expression of $\mathrm{HCV}$ nonstructural proteins including NS2, NS3, NS3/4A, NS4A, NS4B, NS5A and NS5B in HEK293T cells, their ORFs were cloned into the pRKFlag, respectively. The templates expressing HCV proteins were kindly provided by Prof. Ying Zhu (Wuhan University, China). The point mutations were introduced into the NS2 coding region by PCR-mediated mutagenesis with the appropriate primers. For expression of recombinant NS2 in sf9 cells, the ORFs of NS2 and its mutant were constructed into the vector pFastBac HTB-MBP as previously described (Yang et al. 2017). The resulting plasmids were subjected to the Bac-to-Bac baculovirus expression system to express the fusion proteins with an MBP tag at the N-terminus. The EGFP-siRNA was chemically synthesized by Guangzhou RiboBio Co., Ltd., China. All the primers and oligonucleotides used in this study are shown in Supplementary Table S1.

\section{Cell Culture and Transfection}

HEK293T, 293T-NoDice (kindly provided by Prof. Bryan Cullen, Durham, NC, USA) and Huh7.5 cells were maintained in Dulbecco's modified Eagle's medium (DMEM, Gibco) containing 7\% fetal bovine serum (FBS) (Gibco), $100 \mathrm{U} / \mathrm{mL}$ penicillin and $100 \mu \mathrm{g} / \mathrm{mL}$ streptomycin at $37^{\circ} \mathrm{C}$ in an incubator with $5 \% \mathrm{CO}_{2}$. Cells were seeded in 6-well plates and grown overnight to reach $50 \%$ confluence. Before transfection, the medium was changed to DMEM containing without serum and antibiotic. Cells then were transfected with the indicated plasmids by using FuGENE HD Reagent (Roche, Basel, Switzerland) according to the manufacturer's instructions.

\section{Western Blotting}

Cells were harvested in lysis buffer $[50 \mathrm{mmol} / \mathrm{L}$ Tris-HCl (pH 7.4), $150 \mathrm{mmol} / \mathrm{L} \mathrm{NaCl}, 1 \%$ NP40, $0.25 \%$ deoxycholate and a protease inhibitor cocktail (Rhoche)]. Then the lysates were subjected to $15 \%$ SDS-PAGE and Western blotting analysis. The antibodies used in this study are as follow: anti-Tubulin (Proteintech Group, 1:5000), anti-Flag (Proteintech Group, 1:5000) and anti-Myc (Proteintech Group, 1:3000).

\section{Northern Blotting}

Total cellular RNAs were extracted using Trizol reagent (Thermo) according to the manufacturer's instructions. For 
the detection of EGFP mRNA, $5 \mu \mathrm{g}$ of total RNAs were subjected to denatured $1.5 \%$ agarose gels with $2.2 \mathrm{~mol} / \mathrm{L}$ formaldehyde. The separated RNAs were transferred onto the Hybond-A nylon membrane (GE Healthcare), and fixed at $120{ }^{\circ} \mathrm{C}$ for $15 \mathrm{~min}$. Then the membranes were hybridized with DIG-labeled probes in Hybridization Ovens at $65{ }^{\circ} \mathrm{C}$ overnight. Finally, the membranes were incubated with anti-DIG antibody conjugated with alkaline phosphatase and exposed to the luminescent image analyzer LAS4000 (Fuji Film). The probes for detection of EGFP and GAPDH mRNA were complementary to their ORF region of 500-720 nt and 760-1060 nt, respectively. For detection of small RNAs, $20 \mu \mathrm{g}$ of total RNAs were subjected to $7 \mathrm{~mol} / \mathrm{L}$ urea-15\% PAGE and transferred to Hybond-A nylon membrane (GE Healthcare). The membrane was chemically cross-linked in 1-ethly-3-(3dimethylaminopropyl) carbodiimide (EDC) at $60^{\circ} \mathrm{C}$ for $30 \mathrm{~min}$. The DIG-labeled RNA probes targeting EGFP siRNA and U6 were synthesized by Takara.

\section{Expression and Purification of Recombinant Proteins}

The expression and purification of MBP alone and MBPfusion proteins were performed in sf9 cells using baculovirus expression system as previously described (Yang et al. 2017). Briefly, cells were infected with MBP-tagged HCV NS2 expressing baculoviruses for $72 \mathrm{~h}$. Infected cells were resuspended, lysed via sonication and then centrifuged at $11,000 \times g$ for $30 \mathrm{~min}$ to remove debris. The protein in the supernatant was purified using amylose affinity chromatography (New England BioLabs, Ipswich, MA) according to the manufacturer's protocol and then concentrated using Amicon Ultra-15 filters (Millipore, Schwalbach, Germany). All purified proteins were quantified with a bicinchoninic acid (BCA) protein assay Kit (CWBIO, China) and stored at $-80{ }^{\circ} \mathrm{C}$ in aliquots. Proteins were separated on $10 \%$ SDS-PAGE and visualized by Coomassie blue.

\section{Electrophoretic Gel Shift Assay}

We generated 200-nt DIG-labeled dsRNA and 22-nt siRNA via in vitro transcription using DIG RNA labeling mix (Roche). MBP-fusion NS2 or mutant proteins were reacted with DIG-labeled RNAs $(0.2 \mu \mathrm{mol} / \mathrm{L} \quad 200-\mathrm{nt}$ dsRNA or 22-nt siRNA) in a binding buffer [ $50 \mathrm{mmol} / \mathrm{L}$ HEPES (pH 8.0), $15 \mathrm{mmol} / \mathrm{L} \mathrm{NaCl}, 0.5 \mathrm{mmol} / \mathrm{L} \mathrm{MgCl}_{2}$, $10 \%$ glycerol and $1 \mathrm{U}$ of RNase inhibitor (Promega)] at $22{ }^{\circ} \mathrm{C}$ for $45 \mathrm{~min}$; the total volume was $10 \mu \mathrm{L}$. Then the reaction mixtures were separated on $6 \%$ (for dsRNA) or $12 \%$ (for siRNA) TBE-PAGE and transferred to Hybond-A nylon membrane (GE Healthcare). The membranes were incubated for 30 min with anti-DIG antibody conjugated with alkaline phosphatase (Roche).

\section{RNA-IP}

HEK293T cells were lysed in a lysis buffer containing $20 \mathrm{mmol} / \mathrm{L}$ Tris-HCl (pH 7.4), $200 \mathrm{mmol} / \mathrm{L} \quad \mathrm{NaCl}$, $2.5 \mathrm{mmol} / \mathrm{L} \mathrm{MgCl}_{2}, 0.5 \%$ Triton X100, $0.5 \mathrm{U} / \mu \mathrm{L}$ RNase inhibitor (Promega) and a protease inhibitor cocktail (Roche). After centrifugation for $15 \mathrm{~min}$ at $12,000 \times \mathrm{g}$, the supernatant was pre-cleared via incubation with protein-A/ $\mathrm{G}$ agarose beads (Roche) at $4{ }^{\circ} \mathrm{C}$ for $4 \mathrm{~h}$. Then the precleared lysates were incubated with antibodies (anti-Flag, anti-Myc or anti-IgG as a negative control) together with protein-A/G agarose beads (Roche) at $4{ }^{\circ} \mathrm{C}$ for $12 \mathrm{~h}$. The antibody-bound complexes were washed for five times with the same lysis buffer except that $\mathrm{NaCl}$ concentration was raised to $600 \mathrm{mmol} / \mathrm{L}$. Finally, RNAs were extracted using Trizol reagent (Thermo) according to the manufacturer's protocol.

\section{Results}

\section{HCV NS2 has RNAi Suppression Activity in Mammalian Cells}

To identify whether HCV has any nonstructural protein which works as a potential VSR, we screened them via the reversal-of-silencing assay in HEK293T cells (Fig. 1A). In brief, cells were co-transfected with the plasmids encoding EGFP and EGFP-specific shRNA (shEGFP), together with the vectors for divers $\mathrm{HCV}$ nonstructural proteins. NoV B2 (NB2), a well-characterized VSR was used as a positive control. The expression of the HCV-encoded proteins and NB2 were detected by Western blotting with anti-Flag and anti-Myc antibodies (Fig. 1B). At $48 \mathrm{~h}$ post transfection (hpt), the mRNA levels of EGFP were detected by Northern blotting with a digoxigenin (DIG)-labeled RNA probe targeting 500-720 nt of EGFP ORF. EGFP-specific shRNA can effectively eliminate the EGFP transcripts (Fig. 1A, lane 2). As shown in Fig. 1A, HCV NS2 protein can effectively restore the expression of RNAi-silenced EGFP (lane 4). Expectedly, expression of NB2 or genetic ablation of Dicer (NoDice) suppressed shRNA-induced RNAi in HEK293T cells (Fig. 1A, lanes 3 and 11). Importantly, HCV NS2 did not affect the transcription efficiency of EGFP in the absence of shRNA (Fig. 1C), excluding out the possibility that HCV NS2 can directly promote EGFP transcription.

We sought to examine whether the RNAi suppression activity of HCV NS2 is dependent on the protein expression levels. Thus, HEK293T cells were co-transfected with 
A

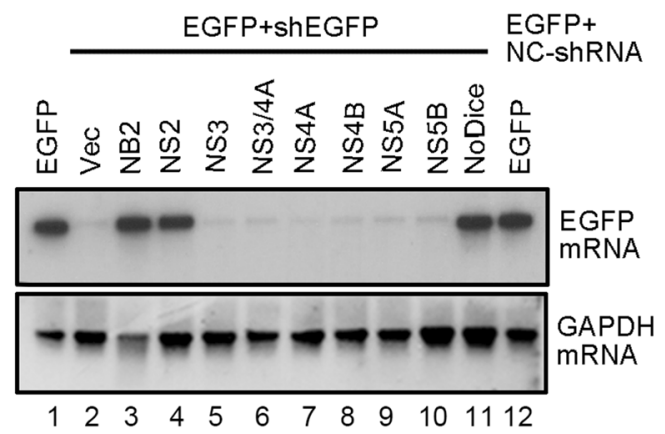

B

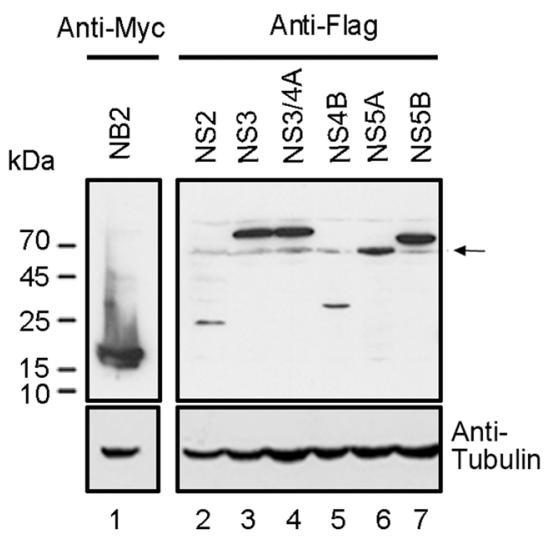

C

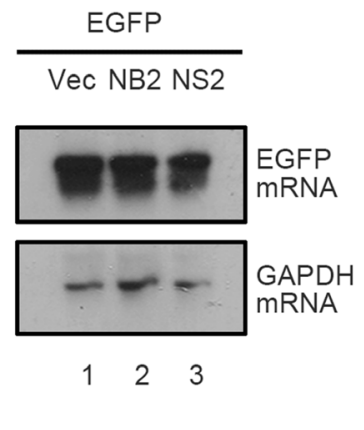

Fig. 1 HCV NS2 contains RNAi suppression activity in mammalian cells. A HEK293T cells were co-transfected with the plasmids encoding EGFP $(0.1 \mu \mathrm{g})$ and EGFP-specific shRNA $(0.3 \mu \mathrm{g})$, together with either empty plasmid or a plasmid encoding $\mathrm{HCV}$ nonstructural protein as indicated or NoV B2 (NB2) (1 $\mu \mathrm{g}$ for each). 293T-NoDice cells and HEK293T cells transfected nonspecificshRNA (NC-shRNA) were used as controls. At $48 \mathrm{hpt}$, total RNAs were extracted and the level of EGFP mRNA was examined via

the plasmids for EGFP and EGFP-specific shRNA, together with the increasing amount of NS2 plasmid as indicated. Our findings showed that the reversal effect of EGFP silencing increased progressively at the mRNA levels, along with the growing amount of NS2 plasmid being transfected (Fig. 2A, 2B). We further examined the VSR activity of HCV NS2 at different time points. Our results showed that the reversal effect of EGFP silencing could be observed at $48 \mathrm{hpt}$ (Fig. 2C), indicating that the VSR activity was dependent on the expression level of $\mathrm{HCV}$ NS2 protein. Taken together, our findings showed that HCV NS2 displays VSR activity in mammalian cells.

\section{HCV NS2 Protein Inhibits Dicer-Mediated siRNA Generation by Sequestrating dsRNA}

Having established that HCV NS2 contains RNAi suppression activity, we sought to examine the detailed mechanism through which HCV NS2 inhibits RNAi. In the process of RNAi, dsRNA/shRNA requires the Dicer-mediated cleavage into siRNA (Maillard et al. 2019). To investigate whether HCV NS2 can inhibit this step, small RNAs harvested from HEK293T cells co-expressing EGFP-specific shRNA together with NS2 were subjected to Northern blotting with a DIG-labeled RNA probe targeting EGFP siRNA produced from shRNA by Dicer. As shown in Fig. 3A, the accumulation of Dicer-cleaved siRNA was reduced in the presence of NS2 compared to that in cells expressing empty vector, indicating that HCV NS2 can suppress Dicer-mediated siRNA production. These results
Northern blotting with DIG-labeled RNA probe targeting the 500-720 nt of EGFP ORF. GAPDH mRNA was used as the loading control. B The expression of HCV proteins was detected by Western blotting. Arrow indicates the unrelated bands in the bot. C HEK293T cells were co-transfected with the plasmid encoding EGFP, together with HCV NS2 or NoV B2 plasmid, respectively. At 48 hpt, EGFP mRNA levels were examined via Northern blotting.

are consistent with the previous findings that HCV NS2 effectively restored shRNA-induced silencing of EGFP transcript in HEK293T cells (Fig. 1A).

In the process of shRNA-induced RNAi, shRNA is cleaved by Dicer into siRNA. After identifying that $\mathrm{HCV}$ NS2 suppressed the siRNA production in cells expressing shRNA, we performed RNA immunoprecipitation (RNAIP) assay to test whether HCV NS2 sequestrated dsRNA in HEK293T cells. Briefly, cells expressing Flag-tagged NS2, Myc-tagged NB2 or empty vector, together with EGFPspecific dsRNA (1-196 nt of EGFP ORF) were lysed and immunoprecipitated with the indicated antibodies, respectively. RNAs extracted from the RNA-IP precipitates were examined via Northern blotting with the RNA probe targeting the 196-nt dsRNA of EGFP. As shown in Fig. 3B, our findings show that NS2 can associate with dsRNA in cells (lane 9).

We sought to examine whether HCV NS2 could directly bind to dsRNA. To this end, we performed gel shift assays by incubating the recombinant MBP-fusion $\mathrm{HCV}$ NS2 (MBP-NS2; supplementary Fig. S1A) and the in vitrotranscribed 200-nt DIG-labeled dsRNA. As shown in Fig. 3C, the mobility of dsRNA was inhibited in a dosedependent manner by the HCV NS2 protein (lanes 4-7), compared to the control reaction with MBP protein alone. Of note, MBP-fusion WhNV B2 (MBP-WB2), another well-characterized VSR that contains the dsRNA-binding activity, was used as the positive control (Fig. 3C, lane 3). Taken together, our findings indicate that HCV NS2 can suppress RNAi by sequestrating dsRNA. 
A

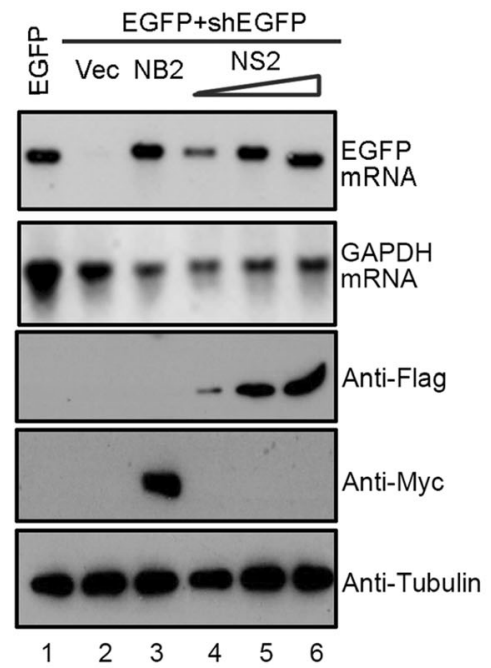

B

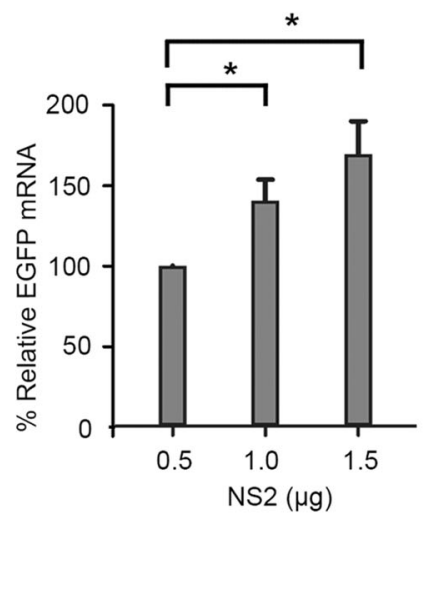

C

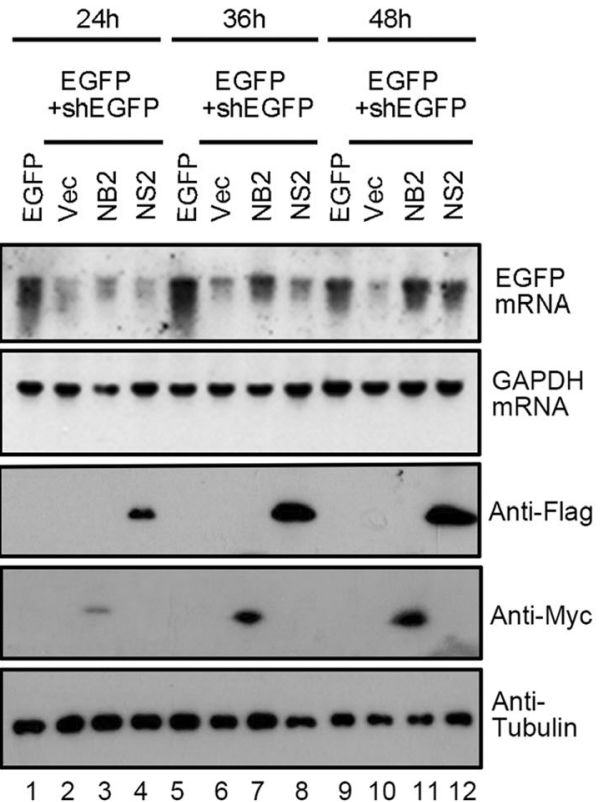

Fig. 2 HCV NS2 suppresses shRNA-induced RNAi in a dosedependent and time-dependent manner in mammalian cells. HEK293T cells were co-transfected with the plasmids encoding EGFP $(0.1 \mu \mathrm{g})$ and EGFP-specific shRNA $(0.3 \mu \mathrm{g})$, together with the increasing amounts of the plasmid encoding HCV NS2 $(0.5 \mu \mathrm{g}$, $1.0 \mu \mathrm{g}$ and $1.5 \mu \mathrm{g}$, respectively). At $48 \mathrm{hpt}$, EGFP mRNA levels were determined by Northern blotting (A). GAPDH mRNA was used as the loading control. The mRNA levels of EGFP normalized to that of GAPDH were quantified with Bio-Rad Quantity One software and were graphed in $(\mathbf{B})$ with that in the presence of the HCV NS2 $(0.5 \mu \mathrm{g})$ defined as $100 \%$. Error bars represent SD values from the

\section{HCV NS2 Suppresses the siRNA-Induced RNAi in Mammalian Cells}

We sought to examine whether HCV NS2 protein can inhibit RNAi induced by siRNA. To this end, HEK293T cells were co-transfected the plasmid for EGFP and the chemically synthesized EGFP-specific siRNA (siEGFP, $50 \mathrm{nmol} / \mathrm{L}$ ), together with the NS2 plasmid. EGFP-specific siRNA induces a significant reduction of EGFP expression (Fig. 4A, lane 2). Our results showed that HCV NS2 efficiently restored the EGFP mRNA levels, indicating that HCV NS2 can inhibit siRNA-induced RNAi in mammalian cells (Fig. 4A, lane 4).

Because HCV NS2 can directly bind to dsRNA, we sought to determine whether NS2 suppressed siRNA-induced RNAi by directly binding to siRNA. To this end, we conducted gel shift assay by incubating MBP-NS2 together with DIG-labeled synthetic 22-nt siRNA. As shown in Fig. 4B, NS2 can directly bind to siRNA in a dose-dependent manner. Taken together, these results indicate that HCV NS2 can suppress RNAi by sequestering siRNA. results of three independent experiments. $* P<0.05$, as measured by two-way ANOVA (GraphPad Prism). Cell lysates were harvested and analyzed by Western blotting with anti-Flag, anti-Myc and antiTubulin antibodies. C HEK293T cells were co-transfected with plasmids encoding EGFP $(0.1 \mu \mathrm{g})$ and EGFP-specific shRNA $(0.3 \mu \mathrm{g})$, together with either empty vector or the plasmid encoding HCV NS2 $(1 \mu \mathrm{g})$ or NB2 $(1 \mu \mathrm{g})$ as indicated. At 24, 36, and $48 \mathrm{hpt}$, total RNAs were extracted and the level of EGFP mRNA was examined by Northern blotting. Cell lysates were harvested and analyzed by Western blotting with anti-Flag, anti-Myc and antiTubulin antibodies.

\section{C184A of HCV NS2 is Critical for the VSR Activity}

After determining the VSR activity of HCV NS2, we sought to identify the critical residues required for its RNAi suppression activity. Thus, we performed multiple sequence alignments of NS2s encoded by multiple HCV isolates (supplementary Fig. S1B). Because the dsRNA/ siRNA-binding and protein dimerization are critical for VSR's activity (Maillard et al. 2019), the positivelycharged and highly conserved residues, including arginine $(\mathrm{R})$, lysine $(\mathrm{K})$, histidine $(\mathrm{H})$, glutamic acid (E) and cysteine $(\mathrm{C})$ were subjected to single-point mutation to alanine (A), and the resulting mutant NS2 proteins were then examined via the reversal-of-silencing assay in HEK293T cells (Fig. 5A-5C). Our findings show that the C184A mutation $\left(\mathrm{NS} 2_{\mathrm{C} 184 \mathrm{~A}}\right)$ significantly suppressed the activity of HCV NS2 to suppress RNAi (Fig. 5C, lane 7 and Fig. 5D, lane 5). Moreover, we further confirmed that the C184 is critical for the VSR activity of HCV NS2 via the reversal-of-silencing assay in Huh7.5 cell line (Fig. 5E), which is usually used for hepatitis $\mathrm{C}$ research. Moreover, we found that C184A abolished the dsRNA- and siRNAbinding activities of HCV NS2 in vitro (Fig. 6A, 6B). 
A

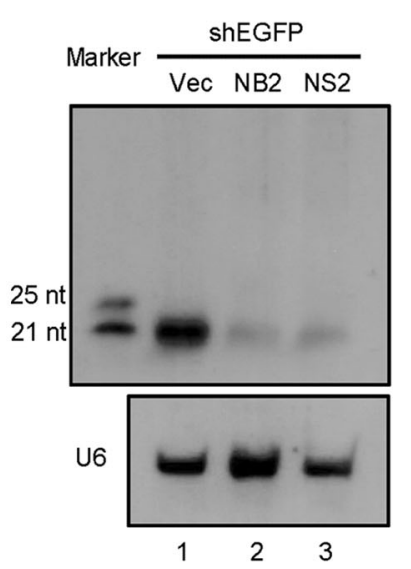

B

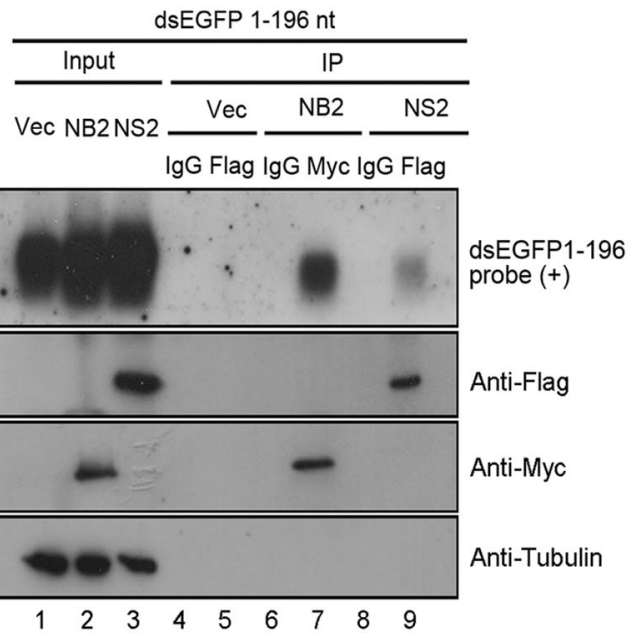

C

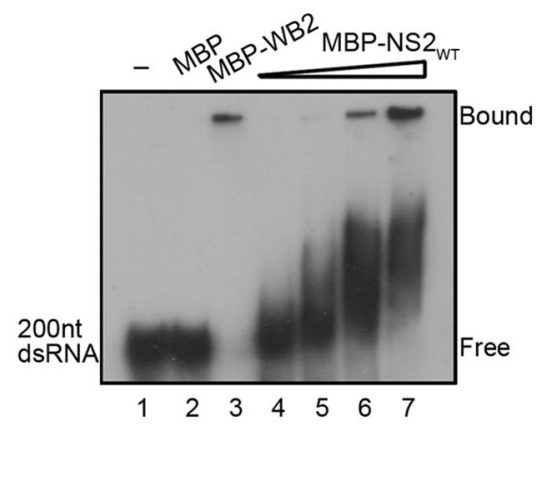

Fig. 3 HCV NS2 binds to dsRNA in cells and in vitro. A HEK293T cells were co-transfected with EGFP specific shRNA $(0.3 \mu \mathrm{g})$ and empty plasmid or a plasmid encoding HCV NS2 or NB2 $(1 \mu \mathrm{g})$. At 48 hpt, total RNAs were extracted for small Northern blotting with either DIG-labeled oligo RNA probe targeting EGFP-shRNA or U6. The synthetic 21- and 25-nt RNAs were used as size markers. B HEK293T cells were transfected with empty plasmid or a plasmid encoding HCV NS2 or NB2 (5 $\mu \mathrm{g}$ for each). At $24 \mathrm{hpt}$, cells were transfected with the dsRNA derived from 1 to $196 \mathrm{nt}$ of EGFP ORF that was transcribed by T7 RNA polymerase in vitro. Then $24 \mathrm{~h}$ later, cell lysates were subjected to RNA-IP with anti-Flag and anti-IgG antibody (as a negative control). Northern blotting was performed to detect precipitated NS2-bound dsRNA by an RNA probe targeting the 1-100 nt of EGFP ORF. NB2-bound dsRNA was used as a positive control (lane 7). C The increasing amount of purified MBP-NS2 protein at concentrations of $0,2,10$, and $15 \mathrm{umol} / \mathrm{L}$ (lanes 4-7) were incubated with $0.2 \mu \mathrm{mol} / \mathrm{L}$ 200-nt DIG-labeled dsRNA at $22{ }^{\circ} \mathrm{C}$ for 45 min. Complexes were separated on $6 \%$ TBE-PAGE.
A

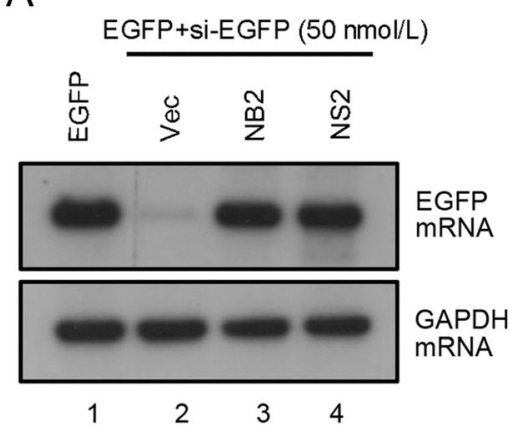

Fig. 4 HCV NS2 suppresses the siRNA-induced RNAi in mammalian cells. A HEK293T cells were co-transfected with a plasmid encoding EGFP $(0.1 \mu \mathrm{g})$ and chemically synthesized EGFP-specific siRNA $(50 \mathrm{nmol} / \mathrm{L})$, together with either empty plasmid or a plasmid encoding HCV NS2 or NB2 (1 $\mu \mathrm{g}$ for each). At $48 \mathrm{hpt}$, total RNAs

Besides, we also found that $\mathrm{NS} 2_{\mathrm{C} 184 \mathrm{~A}}$ failed to suppress the processing of shRNA into siRNA in HEK293T cells (Fig. 6C).

\section{Discussion}

RNAi is an antiviral immune mechanism conserved in both invertebrates and mammals. As a counter-defense to RNAi, viruses evolved to encode VSRs that were identified to

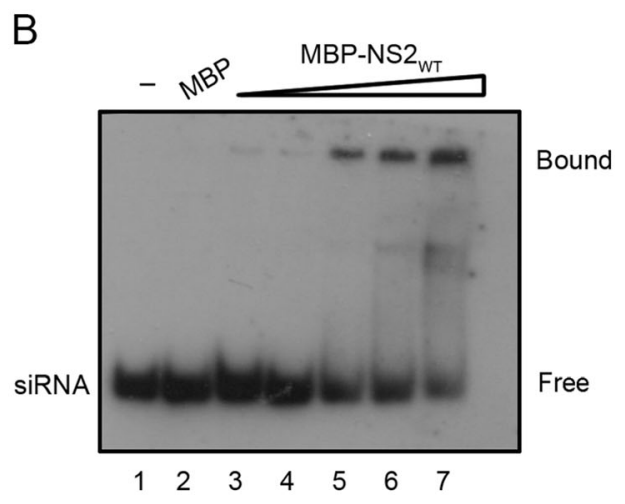

were extracted for Northern blotting. GAPDH mRNA was used as the loading control. B The increasing amount of purified MBP-NS2 protein at concentrations of $0,3,7,11$, and 15 umol/L (lanes 3-7) were incubated with $0.2 \mu \mathrm{mol} / \mathrm{L}$ 22-nt DIG-labeled siRNA at $22{ }^{\circ} \mathrm{C}$ for $45 \mathrm{~min}$. Complexes were separated on $12 \%$ TBE-PAGE.

target different steps of the RNAi pathway. In the present study, we screened the viral nonstructural proteins of $\mathrm{HCV}$ for RNAi suppression activity and found that HCV NS2 protein is a VSR. The gel shift and RNA-IP analyses show that HCV NS2 possesses both dsRNA- and siRNA-binding activities that are required for RNAi suppression. Thus, we speculate that HCV NS2 may function as VSR by shielding virus-derived dsRNA from Dicer cleavage and binding to viral siRNA to interfere with RISC assembly. 
A

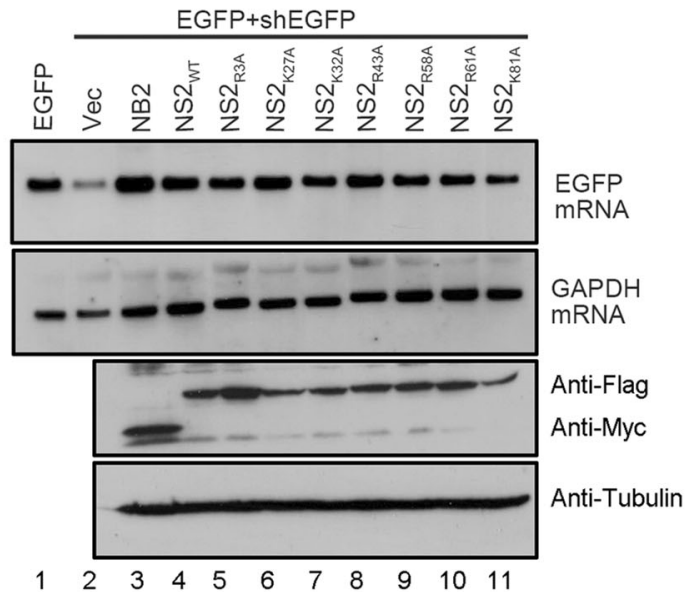

B

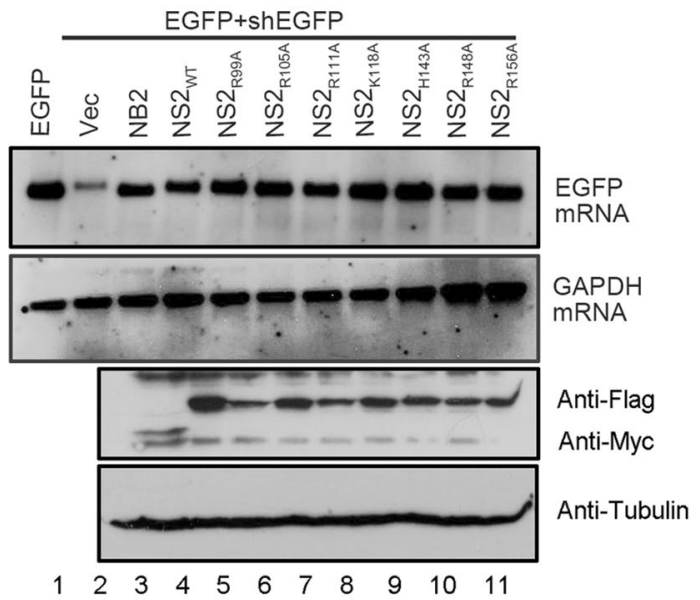

C

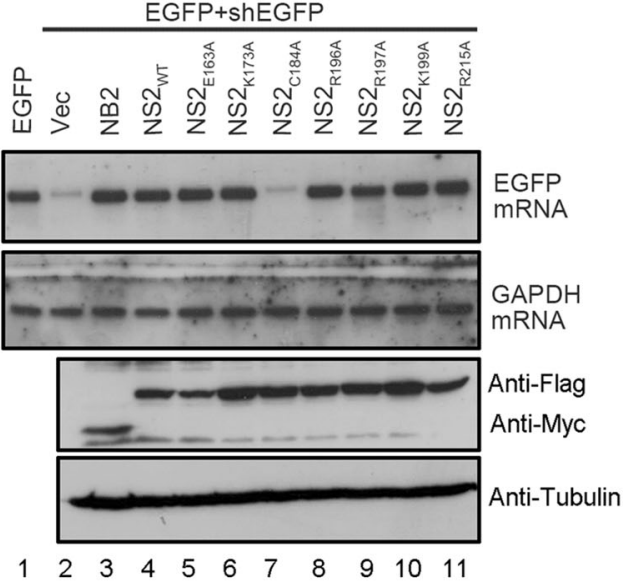

D

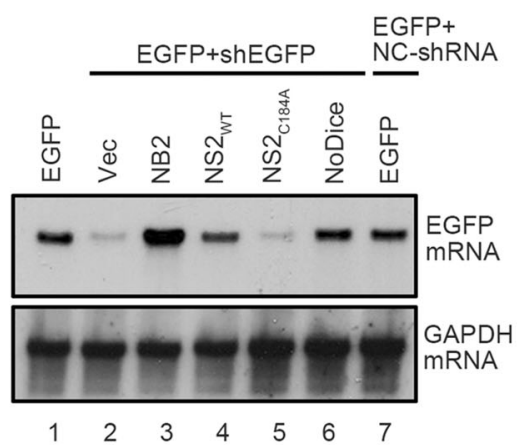

E
Fig. $5 \mathrm{C} 184$ is critical for the VSR activity of HCV NS2. AD HEK293T cells were co-transfected with the plasmids encoding EGFP $(0.1 \mu \mathrm{g})$ and EGFP-specific shRNA $(0.3 \mu \mathrm{g})$, together with either empty vector or the plasmids encoding different HCV NS2 mutants $(1 \mu \mathrm{g}$ each) as indicated, At $48 \mathrm{hpt}$, total RNAs were extracted and the levels of EGFP mRNA were determined via Northern blotting. 293T-NoDice cells were used as a control. GAPDH mRNA was used as the loading control. Cell lysates were also

In addition to NS2, HCV structural proteins core and E2 were also shown to contain RNAi suppression activity by directly targeting the protein components of the RNAi pathway (Chen et al. 2008; Ji et al. 2008). HCV core interacts with Dicer and inhibits the siRNA production, while HCV E2 can block the RISC assembly by interacting with AGO2. Interestingly, we showed that HCV NS2 as a VSR can target the RNA components of the RNAi pathway by directly binding to dsRNA and siRNA. These results indicate that $\mathrm{HCV}$ can suppress antiviral RNAi at different stages by encoding multiple VSRs. This phenomenon can also be found in other RNA viruses, such as EBOV, SARSCoV, HIV-1 and DENV (Bennasser et al. 2005; Karjee et al. 2010; Fabozzi et al. 2011; Aqil et al. 2013; Kakumani et al. 2013; Cui et al. 2015; Kakumani et al. 2015). Thus, subjected to western blotting with anti-Flag, anti-Myc and antiTubulin antibodies. E Huh7.5 cells were co-transfected with the plasmids encoding EGFP $(0.25 \mu \mathrm{g})$ and EGFP-specific shRNA $(0.75 \mu \mathrm{g})$, together with either empty vector or a plasmid encoding $\mathrm{NS} 2$ or $\mathrm{NS} 2_{\mathrm{C} 184 \mathrm{~A}}$ as indicated $(1.5 \mu \mathrm{g}$ each). At $48 \mathrm{hpt}$, total RNAs were extracted and the level of EGFP mRNA was examined via Northern blotting.

encoding multiple VSRs that target different steps of the RNAi pathway may offer advantages in the mode of action required for efficient suppression of RNAi, thereby highlighting the importance of the antiviral RNAi in the hostvirus interactions.

$\mathrm{HCV}$ NS2 protein is a $\sim 23 \mathrm{kDa}$ transmembrane protein that has multiple functions during the $\mathrm{HCV}$ replication. It contains a protease domain that functions as a cysteine protease to catalyze a cleavage between NS2 and NS3 (Pieroni et al. 1997). Besides, NS2 colocalizes with E1, E2 NS3 and NS5A near the core protein and lipid droplet during the virion assembly (Ma et al. 2011). Moreover, NS2 can work as a potent interferon antagonist and apoptosis inhibitor (Erdtmann et al. 2003; Kaukinen et al. 2013). Here we found that HCV NS2 contains an RNAi 

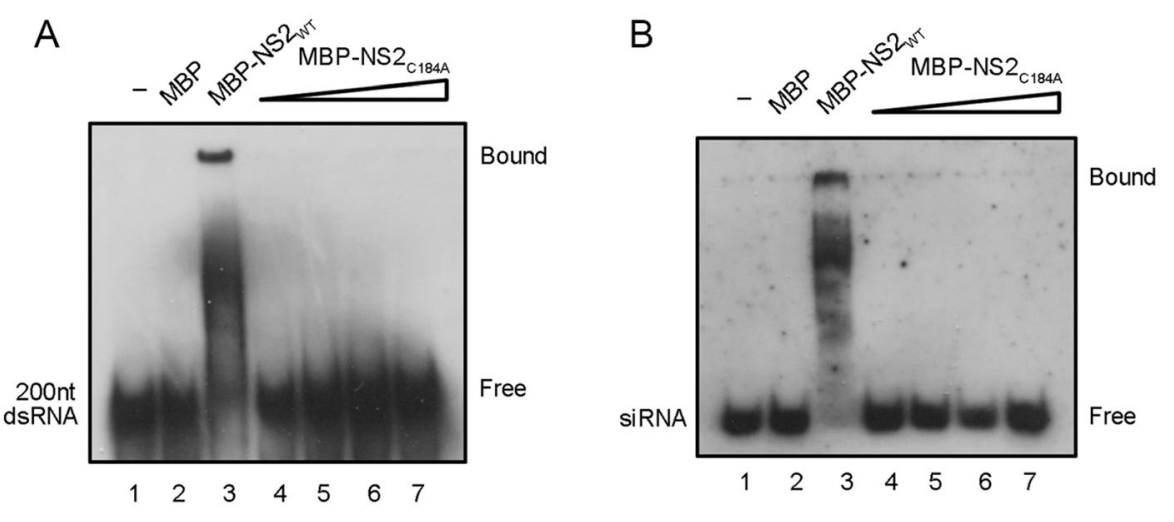

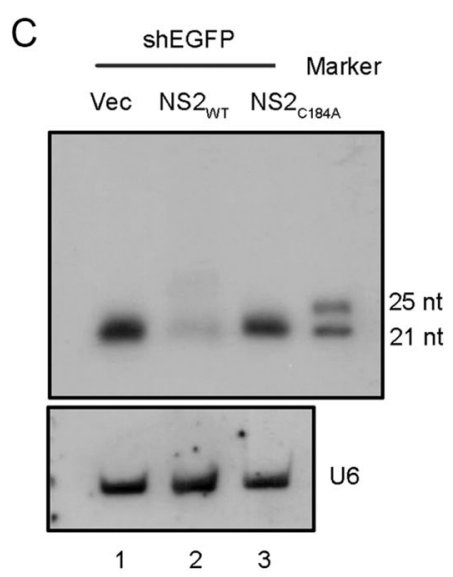

Fig. 6 The dsRNA- and siRNA-binding activities of HCV NS2 are required for its RNAi suppression. MBP-NS2 $2_{\mathrm{WT}}$ and $\mathrm{MBP}-\mathrm{NS} 2_{\mathrm{C} 184 \mathrm{~A}}$ were incubated with $0.2 \mu \mathrm{mol} / \mathrm{L}$ 200-nt DIG-labeled dsRNA (A) or 22-nt siRNA (B) at $22{ }^{\circ} \mathrm{C}$ for $45 \mathrm{~min}$. Complexes were separated on $6 \%$ (A) or 12\% (B) TBE-PAGE, respectively. C HEK293T cells were

suppression activity, adding a novel function to its multiple roles in the viral life cycle. $\mathrm{HCV}$ NS2 is identified to possess dsRNA- and siRNA-binding activities, similar to many other VSRs such as NoV B2 and SARS-CoV nucleocapsid. However, although these VSRs use the same strategy of suppressing RNAi by sequestrating dsRNA and/ or siRNA, they do not share sequence identity or structural conservation, suggesting that VSRs of distinct virus families are evolved independently during the viral evolution.

HCV NS2 forms a dimer with an N-terminal alpha-helical subdomain and a C-terminal antiparallel beta-sheet (Lorenz et al. 2006). In addition, the conserved residues H143, E163 and C184 have been found to important for HCV NS2's dimerization (Lorenz et al. 2006). Our mutational analyses showed that mutation of $\mathrm{C} 184$ abolished the dsRNA- and siRNA-binding activities of HCV NS2 and eliminated its VSR activity, implying the dimerization of HCV NS2 is required for its RNAi suppression activity. Our findings are consistent with the previous observations that dimerization is important for VSR activities of many viruses. For example, EV-A71 3A forms a dimer and disrupting its dimerization blocks the VSR activity (Qiu et al. 2017).

In conclusion, our findings demonstrate that HCV NS2 can act as a VSR by sequestrating dsRNA from Dicer cleavage and interfering with RISC assembly by siRNAbinding. Moreover, these results add HCV NS2 as a new member of HCV-encoded VSRs, which suppressing RNAi by a different mechanism. And it provides insight into the life cycles and virus-host interactions of HCV.

Acknowledgements We wish to thank Prof. Ying Zhu (Wuhan, China) and Prof. Bryan Cullen (Durham, USA) for kindly providing materials. This work was supported by the Strategic Priority Research co-transfected with EGFP specific shRNA $(0.3 \mu \mathrm{g})$ and either the plasmid encoding $\mathrm{NS} 2_{\mathrm{WT}}$ or $\mathrm{NS} 2_{\mathrm{C} 184 \mathrm{~A}}(1 \mu \mathrm{g}$ of each). Total RNAs were subjected to small RNA Northern blotting. U6 was used as the loading control.

Program of Chinese Academy of Sciences (XDB29010300 to X.Z.), the National Natural Science Foundation of China (81873964 to Y.Q., 31670161 to X.Z. and 31800140 to J.M.), the Science and Technology Bureau of Wuhan (2018060401011309 to X.Z.), and the Advanced Customer Cultivation Project of Wuhan National Biosafety Laboratory (2018ACCP-MS11 to Y.Q.). X.Z. is supported by the Newton Advanced Fellowship from the Academy of Medical Sciences, UK (NAF005\1002).

Author Contributions HZ performed the experiments, analyzed the data and drafted the manuscript; QQ, TS, JX, JK and JM helped to perform the experiments; QQ and TS helped to analyze the data; YQ and $\mathrm{XZ}$ designed the experiments, analyzed the data, and finalized the manuscript. All authors approved the final manuscript.

\section{Compliance with Ethics Standards}

Conflict of interest The authors declare that there are no conflict of interest.

Animal and Human Rights Statement This article does not contain any studies with human or animal subjects performed by any of the authors.

\section{References}

Aqil M, Naqvi AR, Bano AS, Jameel S (2013) The HIV-1 Nef protein binds argonaute- 2 and functions as a viral suppressor of RNA interference. PLoS ONE 8:e74472

Bennasser Y, Le SY, Benkirane M, Jeang KT (2005) Evidence that HIV-1 encodes an siRNA and a suppressor of RNA silencing. Immunity 22:607-619

Chen W, Zhang Z, Chen J, Zhang J, Zhang J, Wu Y, Huang Y, Cai X, Huang A (2008) HCV core protein interacts with Dicer to antagonize RNA silencing. Virus Res 133:250-258

Cui L, Wang H, Ji Y, Yang J, Xu S, Huang X, Wang Z, Qin L, Tien P, Zhou X, Guo D, Chen Y (2015) The nucleocapsid protein of 
coronaviruses acts as a viral suppressor of RNA silencing in mammalian cells. J Virol 89:9029-9043

Erdtmann L, Franck N, Lerat H, Le Seyec J, Gilot D, Cannie I, Gripon P, Hibner U, Guguen-Guillouzo C (2003) The hepatitis C virus NS2 protein is an inhibitor of CIDE-B-induced apoptosis. J Biol Chem 278:18256-18264

Fabozzi G, Nabel CS, Dolan MA, Sullivan NJ (2011) Ebolavirus proteins suppress the effects of small interfering RNA by direct interaction with the mammalian RNA interference pathway. J Virol 85:2512-2523

Guo Z, Li Y, Ding SW (2019) Small RNA-based antimicrobial immunity. Nat Rev Immunol 19:31-44

Haasnoot J, de Vries W, Geutjes EJ, Prins M, de Haan P, Berkhout B (2007) The Ebola virus VP35 protein is a suppressor of RNA silencing. PLoS Pathog 3:e86

Horner SM, Gale M Jr (2013) Regulation of hepatic innate immunity by hepatitis C virus. Nat Med 19:879-888

Ji J, Glaser A, Wernli M, Berke JM, Moradpour D, Erb P (2008) Suppression of short interfering RNA-mediated gene silencing by the structural proteins of hepatitis $\mathrm{C}$ virus. J Gen Virol 89:2761-2766

Kakumani PK, Ponia SS, Sood V, Chinnappan M, Banerjea AC, Medigeshi GR, Malhotra P, Mukherjee SK, Bhatnagar RK (2013) Role of RNA interference (RNAi) in dengue virus replication and identification of NS4B as an RNAi suppressor. J Virol 87:8870-8883

Kakumani PK, Rajgokul KS, Ponia SS, Kaur I, Mahanty S, Medigeshi GR, Banerjea AC, Chopra AP, Malhotra P, Mukherjee SK, Bhatnagar RK (2015) Dengue NS3, an RNAi suppressor, modulates the human miRNA pathways through its interacting partner. Biochem J 471:89-99

Karjee S, Minhas A, Sood V, Ponia SS, Banerjea AC, Chow VT, Mukherjee SK, Lal SK (2010) The 7a accessory protein of severe acute respiratory syndrome coronavirus acts as an RNA silencing suppressor. J Virol 84:10395-10401

Kaukinen P, Sillanpaa M, Nousiainen L, Melen K, Julkunen I (2013) Hepatitis C virus NS2 protease inhibits host cell antiviral response by inhibiting IKKepsilon and TBK1 functions. J Med Virol 85:71-82

Li WX, Li H, Lu R, Li F, Dus M, Atkinson P, Brydon EW, Johnson KL, Garcia-Sastre A, Ball LA, Palese P, Ding SW (2004) Interferon antagonist proteins of influenza and vaccinia viruses are suppressors of RNA silencing. Proc Natl Acad Sci USA 101:1350-1355

Li Y, Lu J, Han Y, Fan X, Ding SW (2013) RNA interference functions as an antiviral immunity mechanism in mammals. Science 342:231-234

Li Y, Basavappa M, Lu J, Dong S, Cronkite DA, Prior JT, Reinecker HC, Hertzog P, Han Y, Li WX, Cheloufi S, Karginov FV, Ding SW, Jeffrey KL (2016) Induction and suppression of antiviral RNA interference by influenza A virus in mammalian cells. Nat Microbiol 2:16250
Lorenz IC, Marcotrigiano J, Dentzer TG, Rice CM (2006) Structure of the catalytic domain of the hepatitis $\mathrm{C}$ virus NS2-3 protease. Nature 442:831-835

Ma Y, Anantpadma M, Timpe JM, Shanmugam S, Singh SM, Lemon SM, Yi M (2011) Hepatitis C virus NS2 protein serves as a scaffold for virus assembly by interacting with both structural and nonstructural proteins. J Virol 85:86-97

Maillard PV, Ciaudo C, Marchais A, Li Y, Jay F, Ding SW, Voinnet O (2013) Antiviral RNA interference in mammalian cells. Science 342:235-238

Maillard PV, van der Veen AG, Poirier EZ, Reis ESC (2019) Slicing and dicing viruses: antiviral RNA interference in mammals. EMBO J 38:e100941

Nayak A, Kim DY, Trnka MJ, Kerr CH, Lidsky PV, Stanley DJ, Rivera BM, Li KH, Burlingame AL, Jan E, Frydman J, Gross JD, Andino R (2018) A viral protein restricts drosophila RNAi immunity by regulating argonaute activity and stability. Cell Host Microbe 24(542-557):e549

Paboriboune P, Vial T, Sitbounlang P, Bertani S, Trepo C, Deny P, Babin FX, Steenkeste N, Pineau P, Deharo E (2018) Hepatitis C in Laos: a 7-year retrospective study on 1765 patients. Virol Sin 33:295-303

Pieroni L, Santolini E, Fipaldini C, Pacini L, Migliaccio G, La Monica N (1997) In vitro study of the NS2-3 protease of hepatitis C virus. J Virol 71:6373-6380

Qi N, Cai D, Qiu Y, Xie J, Wang Z, Si J, Zhang J, Zhou X, Hu Y (2011) RNA binding by a novel helical fold of b2 protein from wuhan nodavirus mediates the suppression of RNA interference and promotes b2 dimerization. J Virol 85:9543-9554

Qi N, Zhang L, Qiu Y, Wang Z, Si J, Liu Y, Xiang X, Xie J, Qin CF, Zhou X, Hu Y (2012) Targeting of dicer-2 and RNA by a viral RNA silencing suppressor in Drosophila cells. J Virol 86:5763-5773

Qiu Y, Xu Y, Zhang Y, Zhou H, Deng YQ, Li XF, Miao M, Zhang Q, Zhong B, Hu Y, Zhang FC, Wu L, Qin CF, Zhou X (2017) Human virus-derived small RNAs can confer antiviral immunity in mammals. Immunity 46(992-1004):e1005

Sullivan CS, Ganem D (2005) A virus-encoded inhibitor that blocks RNA interference in mammalian cells. J Virol 79:7371-7379

Wu Q, Wang X, Ding SW (2010) Viral suppressors of RNA-based viral immunity: host targets. Cell Host Microbe 8:12-15

Xu YP, Qiu Y, Zhang B, Chen G, Chen Q, Wang M, Mo F, Xu J, Wu J, Zhang RR, Cheng ML, Zhang NN, Lyu B, Zhu WL, Wu MH, Ye Q, Zhang D, Man JH, Li XF, Cui J, Xu Z, Hu B, Zhou X, Qin CF (2019) Zika virus infection induces RNAi-mediated antiviral immunity in human neural progenitors and brain organoids. Cell Res 29:265-273

Yang J, Qian Q, Li T-F, Yang X, Won SJ, Zhou X (2017) Cypovirus capsid protein VP5 has nucleoside triphosphatase activity. Virol Sin 32:328-330

Yin P, Li Y, Zhou L, Zhang L (2018) NAP1L1 regulates hepatitis C virus entry and interacts with NS3. Virol Sin 33:205-208 